MS36-P07

\section{Coordination polymers of group 1 and 2 metals with a dimeric capsular zwitterionic pyridinecarboxylate}

\author{
Antti Tiihonen ${ }^{1}$, Manu Lahtinen ${ }^{1}$ \\ 1. Department of Chemistry, University of Jyvaskyla, Jyvaskyla, \\ Finland \\ email: antti.j.tiihonen@jyu.fi
}

Coordination polymers with alkali and alkaline earth metals are not unheard of, but those implementing zwitterionic pyridinecarboxylates are very scarce, with only three records in the CCDC database [1-3]. Tripodal zwitterionic pyridinecarboxylate ligand with a systematic name 1,1 ',1"'-((2,4,6-trimethylbenzene-1,3,5-triyl)tris(methylene))tris(pyridin-1-ium-4-carboxylate) (TTTPC), forms stable capsular dimers in water by enclosing halides with sufficiently large ionic radius, especially bromide and iodide $\left(\left[\mathbf{T T T P C} \mathbf{C}_{2} \mathbf{X}\right]^{-}\right)$. When completely zwitterionic the ligand has neutral net charge, and the enclosed halide ion makes the charge of the capsule one negative. This charge can be balanced by an alkali or alkaline earth metal coordinated to the carboxylate moiety on the outer rim of the capsule forming a 1D polymer with potassium ((KITTTP$\left.\left.\mathbf{C}_{2} \mathbf{X}\right]\right)_{n}$ ) and a 2D polymer with barium, the $2^{+}$charge of the latter metal balanced by an additional halide ((Ba[TTTP$\left.\left.\mathbf{C}_{2} \mathbf{X}\right] \mathbf{X}\right)_{\mathbf{n}}$ ). Both polymers are highly hydrated systems and hydrogen bond networks of water molecules occupy the space between polymeric moieties. Additionally, ammonium as a pseudo-alkali metal functions exactly as potassium, and forms a $0 \mathrm{D}$ hydrogen bonded coordination polymer $\left(\mathbf{N H}_{4}\left[\mathbf{T T T P C} \mathbf{C}_{2} \mathbf{X}\right]\right)$ that mimics the aforementioned 1D polymeric system with an isostructural hydrogen bonded water network. Potassium has a coordination number 7 with four monodentate carboxylates and one bidentate carboxylate in addition to one coordinated water molecule in its first coordination sphere. Barium has a coordination number 9 with an additional bidentate carboxylate. Potassium system closely assimilates the $2 \mathrm{D}$ barium polymer, but must be classified as a $1 \mathrm{D}$ polymer, because of the lack of direct coordination to the metal, having only a hydrogen bond to the coordinated water instead. Mean M-O coordination bond lengths of monodentate carboxylates are $2.779(\mathrm{~K})$ and $2.774(\mathrm{Ba})$ angstroms, and bidentate bond lengths respectively $2.896(\mathrm{~K})$ and $2.894(\mathrm{Ba})$ angstroms. All polymers crystallize in the triclinic space group P-1, and their structures have been determined using single crystal X-ray diffraction. Further studies with other metals in similar systems are underway.
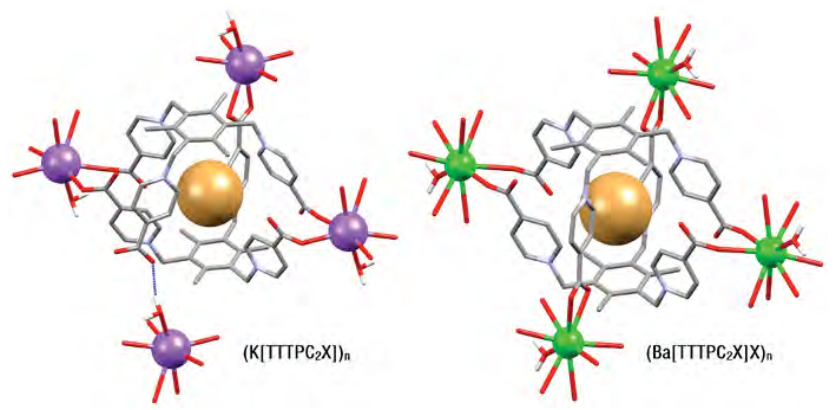

References:

[1] Lin, H. \& He Y.-H. (2007). Acta Cryst. E63, m3204-m3205.

[2] Hao, X.-L. et al. (2014). Inorg. Chem. Commun. 41, 19-24.

[3] Huang, Y.-Q. et al. (2014). Inorg. Chim. Acta. 421, 318-325.

Keywords: Coordination polymers, hydrogen bonding, zwitterions 